jcrpe-2018-0182.R2

Case Report

\title{
Efficiency of Single Dose of Tolvaptan Treatment During the Triphasic Episode After Surgery for Craniopharyngioma Gürbüz F et al. Efficiency of Single Dose Tolvaptan
}

Fatih Gürbüz, Mehmet Taştan, İhsan Turan, Bilgin Yüksel

Çukurova University Faculty of Medicine, Department of Pediatric Endocrinology, Adana, Turkey

\section{Corresponding author}

Fatih Gürbüz MD, Çukurova University Faculty of Medicine, Department of Pediatric Endocrinology, Adana, Turkey

Tel: +903223386060,+905057077366

E-mail: fggurbuz@yahoo.com

ORCID number: 0000-0003-2160-9838

Conflict of interest: None declared

Received: 13.07 .2018

Accepted: 25.09 .2018

\section{What is already known on this topic?}

Hyponatremia is caused by the combination of excess ADH-induced water retention and secondary solute loss in patients with inappropriate antidiuretic hormone syndrome. Vaptans, arginine-vasopressin receptor antagonists are alternatively used for SIADH in adults. In children, vaptan treatment has not been approved for SIADH.

\section{What this study adds?}

Here, we report a successful tolvaptan treatment in a child with SIADH. Furthermore, we report that only one dose of tolvaptan in triphasic episode is sufficient efficiency. This data is the first child patient from Turkey.

\begin{abstract}
Inappropriate ADH syndrome (SIADH) may develop after intracranial surgery. SIADH in pediatric age is mostly seen in intracranial mass and not only after surgery. The management of these clinical conditions in patients is important for the clinical course of the patient. Fluid restriction is standard therapy in SIADH. Resistant hyponatremic pattern may be encountered in some cases. Vaptans have recently started to be used in hyponatremia due to SIADH. A patient with SIADH of triphasic episode presented to our clinical practice after surgery of craniopharyngioma. Resistant hyponatremia was not resolved despite fluid restriction and hypertonic saline support. The patient responded to single dose of tolvaptan fast and well and thus the clinical condition of the patient was successfully under control. There is inadequate data about tolvaptan treatment in pediatric patients. Our case report will contribute to the literature in this regard.
\end{abstract}

Key words: inappropriate ADH syndrome, tolvaptan, children

\section{Introduction}

The syndrome of inappropriate antidiuretic hormone secretion (SIADH) is a disorder of impaired water excretion caused by the insufficiency to suppress the secretion of antidiuretic hormone (ADH) (1). SIADH is a major situation and one of the causes of hyponatremia in hospitalized cases (2). The etiology of SIADH involves excess $\mathrm{ADH}$ production due to cranial surgery, malignancies, meningitis-encephalitis, hemorrhage, other cerebral pathologies, pulmonary malignancies and drugs $(2,3)$.

In clinical practice, common and gold standard approach to SIADH is fluid restriction (4-6). In more severe forms that also show neurological symptoms, administration of hyperosmolar saline combined with furosemide may be required. Additionally, underlying etiology should be treated if possible (3, 5, 6-10). However, these treatments may be inadequate for some patients with SIADH.

In the last decade, a new alternative treatment option for SIADH named vaptans has become available (8). Vaptans are arginine-vasopressin receptor antagonists, thus prevent insertion of aquaporin 2 water channels in the apical membrane promoting reabsorption of water and results in excretion of diluted urine $(3,5,11)$. Tolvaptan is a kind of vaptan and is a selective $\mathrm{V} 2$ receptor antagonist, whereas conivaptan is a non-selective $\mathrm{V} 1 / \mathrm{V} 2$ receptor antagonist $(3,5,6,10)$. Conivaptan is the US Food and Drug Administration (FDA) approved 
for hypervolemic (nephrotic syndrome, cardiac failure and cirrhosis) or euvolemic hyponatremia (SIADH) treatment in adults but not in children (10). Peters et al. (10) described the first successful treatment of conivaptan for a pediatric refractory SIADH patient and mentioned that conivaptan played a key role in the management of their case with SIADH and no adverse effects had developed (10).

Tolvaptan is approved by the FDA since 2009 for adults and successfully used in the treatment of hyponatremia due to SIADH and autosomal dominant polycystic kidney diseases $(1,9,11,12)$. Data on the safety, efficacy and optimal dose of tolvaptan in pediatric patients are limited. There are rare case reports about tolvaptan therapy for SIADH in childhood $(3,5,6,13)$. Likewise, there is scarce data with tolvaptan therapy used to treat hypervolemic hyponatremia such as in cardiac failure and nephrotic syndrome in children (14-17). Tolvaptan treatment has been used successfully for 3 children with SIADH (ROHHAD syndrome, large sellar-suprasellar tumor and surgery of astrocytoma, respectively) (5), for a patient with intracranial lymphoma (3), and a child with nephrotic syndrome (17). Again, 28 pediatric cases with cardiac failure patients who were treated with tolvaptan have been reported (15). It has been noted that tolvaptan therapy is effective, safe and well tolerated in hyponatremia in all these case series.

Here, we report the first pediatric case of severe and symptomatic hyponatremia due to SIADH, successfully treated with single dose tolvaptan.

\section{Case Report}

A 13-year-old girl was referred to our practice because of possible endocrine problems due to craniopharyngioma with a 3-week history of headache and reduction in vision. She was the third child of nonrelated parents. Her birth history was unremarkable. On physical examination, her height was $150.8 \mathrm{~cm}[-1.19$ standard deviation (SD)] and her weight was $60.2 \mathrm{~kg}(1.23 \mathrm{SD})$. General examination was normal except right eye exotropia and reduction in vision on the right.

No endocrine abnormalities were detected before the craniopharyngioma operation (Table 1). On the postoperative first day, dexamethasone treatment for brain-associated surgery was started by the neurosurgeon; therefore no additional steroid treatment was received for central adrenal insufficiency. Furthermore, the patient had polyuria $(5.6 \mathrm{ml} / \mathrm{kg} / \mathrm{h}$ ), plasma sodium was $146 \mathrm{mmol} / \mathrm{L}$ (reference range 135-145), plasma osmolality was $303 \mathrm{mOsm} / \mathrm{kgH}_{2} \mathrm{O}$ and urinary density was 1002 . Desmopressin acetate $(0.1 \mu \mathrm{g} / \mathrm{kg} / \mathrm{day}$, melt form) treatment was started for diagnosed diabetes insipidus (DI). Desmopressin treatment improved polyuria and plasma sodium concentration. On the postoperative 4 th day, levothyroxine $(100 \mu \mathrm{g} /$ day $)$ replacement therapy was started for central hypothyroidism. The patient developed hyponatremia, which started on postoperative day 4 and gradually became deeper. On the postoperative 5th day, urinary output of the patient decreased to $0.7 \mathrm{ml} / \mathrm{kg} / \mathrm{h}$. Evaluation of the laboratory findings (plasma sodium $128 \mathrm{mmol} / \mathrm{L}$, plasma osmolality $267 \mathrm{mOsm} / \mathrm{kgH}_{2} \mathrm{O}$, urinary density 1039) led to the diagnosis of SIADH. Plasma copeptin/ADH levels could not be measured due to laboratory inadequacy. This suggests that SIADH was the second stage of the triphasic condition after cranial surgery. First, fluid restriction was administered (total $800 \mathrm{ml} / \mathrm{m}^{2} /$ day) and desmopressin treatment was discontinued. Despite fluid restriction for 4 days, the patient's blood sodium levels decreased to $118 \mathrm{mmol} / \mathrm{L}$ and urine density was 1039 . Hypertonic saline therapy ( 3 percent saline to raise the serum sodium by $10 \mathrm{mEq} / \mathrm{L}$ ) was also added due to persistence of hyponatremia. However, SIADH could not be controlled and severe hyponatremia continued. In addition, the patient's condition began to worsen and mild loss of consciousness occurred. Therefore, we decided to start low-dose oral tolvaptan treatment $(0.13 \mathrm{mg} / \mathrm{kg} / \mathrm{day})$ on the postoperative 8th day. Written consent form was obtained from the parents for the use of tolvaptan. One hour after oral intake of Tolvaptan, the urine output and plasma sodium levels of the patient began to gradually increase. Urinary output increased to $8.1 \mathrm{ml} / \mathrm{kg} / \mathrm{h}$, urinary density was reduced to 1001 . One dose of tolvaptan administered to the patient was sufficient to control SIADH, no further treatment was given. Moreover, desmopressin treatment was restarted because of the development of diabetes insipidus 42 hours after the administration of tolvaptan (plasma sodium $138 \mathrm{mmo} / \mathrm{L}$, plasma osmolality $296 \mathrm{mOsm} / \mathrm{kgH}_{2} \mathrm{O}$, urinary output $6.6 \mathrm{ml} / \mathrm{kg} / \mathrm{h}$ and urinary density 1002). In the follow-up, persistent diabetes insipidus was observed, requiring desmopressin (Figure 1).

\section{Discussion}

Here we report successful Tolvaptan administration in a patient who developed severe and uncontrolled hyponatremia due to SIADH. In our knowledge, this is the first child patient with a successful Tolvaptan experience reported from Turkey.

Hypothalamus and/or tract damage due to neurosurgery or trauma may frequently result in a typical triphasic response (18-20). First, transient diabetes insipidus, beginning within 24 hours and lasting 4 to 5 days, by reason of reflex inhibition of ADH release due to hypothalamic dysfunction. Following that, on days 6-10, a transient SIADH caused by stored ADH release from the degenerated posterior pituitary. And finally, diabetes insipidus again, after the posterior pituitary stores are consumed. This third phase, diabetes insipidus, may be permanent or transient $(19,20)$. Our patient also had this triphasic response. 
There are two different approaches to managing central diabetes insipidus in cranial surgical patients. The first approach is employing fluids and avoiding the use of vasopressin. It may be particularly useful for managing acute postoperative diabetes insipidus in young children. Vasopressin therapy may mask the emergence of the SIAD phase of the triple phase neurohypophyseal response to neurosurgical injury (21). The other approach is treating with vasopressin. Our patient was 13 -years-old with a weight of $60.2 \mathrm{~kg}$, almost a young adult weight. For this reason, we preferred to use vasopressin for the DI, and didn't observe the masking of the emergence of the SIAD when DI was thought to be able to last 4 to 5 days $(19,20)$.

Also, longer duration of surgery is an associated factor for the triphasic response (22). Our patient had a surgery that lasted about 10.5 hours. But in the second phase, transient SIADH, the patient developed severe and uncontrollable symptomatic hyponatremia. For this reason, even though we knew that this second phase was temporary, we had to intervene due to severe hyponatremia, resistant to fluid restriction and hypertonic saline therapy. And thus, vaptans were the most appropriate choice as an alternative treatment. For the patient we preferred to use tolvaptan which is a selective V2 receptor antagonist, as it may be more effective and cause less side effects. Resistant hyponatremia improved dramatically with a single low-dose tolvaptan administration to the patient.

In a recent article, tolvaptan treatment on 2 infants with non-improving hyponatremia due to SIADH has been mentioned. The treatment was initiated at a dose of $0.8 \mathrm{mg} / \mathrm{kg} / \mathrm{day}$, and the dose was reduced to $0.22 \mathrm{mg} / \mathrm{kg} / \mathrm{day}$ upon the onset of hypernatremia on the second day of treatment in one infant. Tolvaptan was used for 7 months in one of the infants and for 13 months in the other, without any problems (6). In our patient, a single dose of tolvaptan was sufficient, and in our patient, even desmopressin was started due to the development of hypernatremia and conversion of the case to DI. In another article, tolvaptan treatment was given to 3 SIADH patients aged between 4 and 7 years, at a dose of 0.05-0.3 mg/kg/day, and low dose tolvaptan treatment was continued for as long as 3-4 years without complications (5). A long-term therapy such as this is an important emphasis on the safety of the drug.

In fact, this SIADH condition in the patient was temporary and would return to diabetes insipidus, which is the last phase, spontaneously. However, the clinical condition of the patient was too severe to wait for this spontaneous change. Therefore, we accelerated the passage to the third phase with tolvaptan therapy. And finally, 42 hours after tolvaptan, desmopressin treatment for diabetes insipidus was started. No side effects were observed in our patient in terms of tolvaptan.

For tolvaptan, onset of action for aquaretic and sodium increasing effects is 2 to 4 hours, and the peak effect is 4 to 8 hours (23). Willemsen et al. (3) mentioned starting at a low dose to avoid rapid correction of hyponatremia. Furthermore, in another article Peters et al. (10) noted the correction of hyponatremia on the first day after conivaptan was given. In our patient, the aqauretic effect of tolvaptan started to emerge about 1 hour after drug ingestion, even though a low dose was used. This was a fast and immediate effect after a single dose tolvaptan treatment. Therefore, patients need to be closely followed up from the first hour in terms of both the urine output and the increase in their serum sodium levels.

The use of tolvaptan or conivaptan therapy in childhood has still not been approved by FDA or the European Medicines Agency (EMA). The most important reason for this is that there is not enough clinical experience in terms of safety and effectiveness in children. We observed a successful treatment with tolvaptan in a pediatric patient who suffered from SIADH. We report that only one dose of tolvaptan in the triphasic episode has sufficient efficiency. As with this data, new clinical experiences will contribute in this regard.

\section{References}

1. Tzoulis P, Carr H, Bagkeris E, Bouloux PM. Improving care and outcomes of inpatients with syndrome of inappropriate antidiuresis (SIAD): a prospective intervention study of intensive endocrine input vs. routine care. Endocrine, 2017;55(2):539-546.

2. Zieg J. Evaluation and management of hyponatraemia in children. Acta Paediatr. 2014;103(10):10271034.

3. Willemsen RH, Delgado-Carballar V, Elleri D, Thankamony A, Burke GA, Nicholson JC, et al. Tolvaptan use during hyperhydration in paediatric intracranial lymphoma with SIADH. Endocrinol Diabetes Metab Case Rep. 2016;2016. pii: 16-0066. Epub 2016 Nov 1.

4. Verbalis JG, Grossman A, Hoybye C, Runkle I. Review and analysis of differing regulatory indications and expert panel guidelines for the treatment of hyponatremia. Curr Med Res Opin. 2014;30(7):1201-1207.

5. Tuli G, Tessaris D, Einaudi S, De Sanctis L, Matarazzo P. Tolvaptan Treatment in Children with Chronic Hyponatremia due to Inappropriate Antidiuretic Hormone Secretion: A Report of Three Cases. J Clin Res Pediatr Endocrinol. 2017;9(3):288-292. 
6. Marx-Berger D, Milford DV, Bandhakavi M, Van't Hoff W, Kleta R, Dattani M, et al. Tolvaptan is successful in treating inappropriate antidiuretic hormone secretion in infants. Acta Paediatr. 2016;105(7):334337.

7. Moritz ML, Ayus JC. Prevention of hospital-acquired hyponatremia: a case for using isotonic saline. Pediatrics. 2003;111(2):227-230.

8. Aditya S, Rattan A. Vaptans: A new option in the management of hyponatremia. International journal of applied \& basic medical research. 2012;2(2):77-83.

9. Peri A. Clinical review: the use of vaptans in clinical endocrinology. J Clin Endocrinol Metab. 2013;98(4):1321-1332.

10. Peters S, Kuhn R, Gardner B, Bernard P. Use of conivaptan for refractory syndrome of inappropriate secretion of antidiuretic hormone in a pediatric patient. Pediatr Emerg Care. 2013;29(2):230-232.

11. Decaux G, Soupart A, Vassart G. Non-peptide arginine-vasopressin antagonists: the vaptans. Lancet (London, England). 2008;371(9624):1624-1632.

12. Muto S, Kawano H, Higashihara E, Narita I, Ubara Y, Matsuzaki T, Ouyang J, Torres VE, Horie S. The effect of tolvaptan on autosomal dominant polycystic kidney disease patients: a subgroup analysis of the Japanese patient subset from TEMPO 3:4 trial. Clin Exp Nephrol. 2015;19(5):867-877.

13. Kraayvanger L, Latza J, Vockelmann C, Berlit P, Weber R. Tolvaptan treatment of severe stroke-like symptoms and bilateral subcortical diffusion restriction due to syndrome of inappropriate secretion of ADH after polytrauma. J Neurol. 2014;261(7):1436-1438.

14. Higashi K, Murakami T, Ishikawa Y, Itoi T, Ohuchi H, Kodama Y, et al. Efficacy and safety of tolvaptan for pediatric patients with congestive heart failure. Multicenter survey in the working group of the Japanese Society of PEdiatric Circulation and Hemodynamics (J-SPECH). International journal of cardiology. 2016;205:37-42.

15. Regen RB, Gonzalez A, Zawodniak K, Leonard D, Quigley R, Barnes AP, et al. Tolvaptan increases serum sodium in pediatric patients with heart failure. Pediatr Cardiol. 2013;34(6):1463-1468.

16. Horibata Y, Murakami T, Niwa K. Effect of the oral vasopressin receptor antagonist tolvaptan on congestive cardiac failure in a child with restrictive cardiomyopathy. Cardiol Young. 2014;24(1):155-157. 17. Shimizu M, Ishikawa S, Yachi Y, Muraoka M, Tasaki Y, Iwasaki H, et al. Tolvaptan therapy for massive edema in a patient with nephrotic syndrome. Pediatr Nephrol. 2014;29(5):915-917.

18. Ghirardello S, Hopper N, Albanese A, Maghnie M. Diabetes insipidus in craniopharyngioma:

postoperative management of water and electrolyte disorders. J Pediatr Endocrinol Metab. 2006;19 Suppl 1:413421.

19. Hoorn EJ, Zietse R. Water balance disorders after neurosurgery: the triphasic response revisited. NDT Plus. 2010;3(1):42-44.

20. Olson BR, Gumowski J, Rubino D, Oldfield EH. Pathophysiology of hyponatremia after transsphenoidal pituitary surgery. J Neurosurg. 1997;87(4):499-507.

21. Joseph A. Majzoub LJM, Abhinash Srivatsa. Disorders of the Posterior Pituitary. In: Sperling MA (eds). Pediatric Endocrinology, Fourth Edition. Philadelphia, Elsevier Saunders, 2014; 405-443.

22. Finken MJ, Zwaveling-Soonawala N, Walenkamp MJ, Vulsma T, van Trotsenburg AS, Rotteveel J. Frequent occurrence of the triphasic response (diabetes insipidus/hyponatremia/diabetes insipidus) after surgery for craniopharyngioma in childhood. Horm Res Paediatr. 2011;76(1):22-26.

23. https://www.uptodate.com/contents/tolvaptan-drug-information?source=see_link

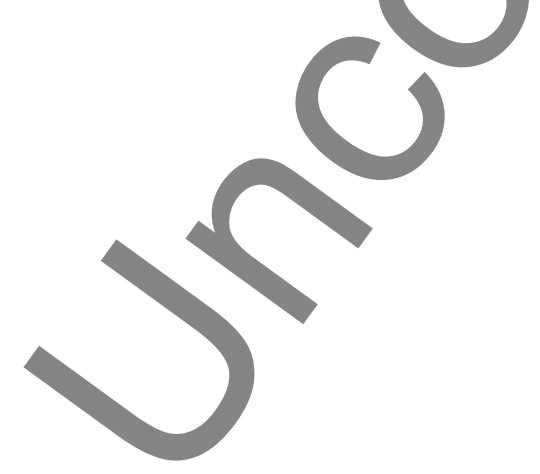


Table 1. Laboratory findings of the patient Normal range

Free T4 (0.67-1.12 ng/dL) TSH (0.5-5.5 mIU/L)

IGF-1 (192-568 ng/mL)

Prolactin $(3.3-26.7 \mathrm{ng} / \mathrm{mL})$

ACTH $(10-50 \mathrm{pg} / \mathrm{mL})$

Cortisol $(6,7-22.6 \mu \mathrm{g} / \mathrm{dL})$

FSH (1.5-12.8 $\mathrm{mIU} / \mathrm{mL})$

LH (0.10-12.0 $\mathrm{mIU} / \mathrm{mL})$

Estradiol $(7-60 \mathrm{pg} / \mathrm{mL})$

Glucose (60-100 mg/dL)

BUN (5-22 mg/dL)

Creatinine (0.3-1 mg/dL)

Serum uric acid (1.9-5.4 mg/dL)

Urine Sodium (mmol/L)

AST (15-40 U/L)

ALT (8-39 U/L)

Preoperative

0.99

1.00

146.1

5.92

19.5

15.98

3.49

1.83

48

88

11

0.51

$\mathrm{NA}$

25

24
Postoperative

0.47

0.002

57.1

4.43

7.42

4.86

0.08

0.02

$<5$

83

9

0.53

1.1

106

22

NA: not available

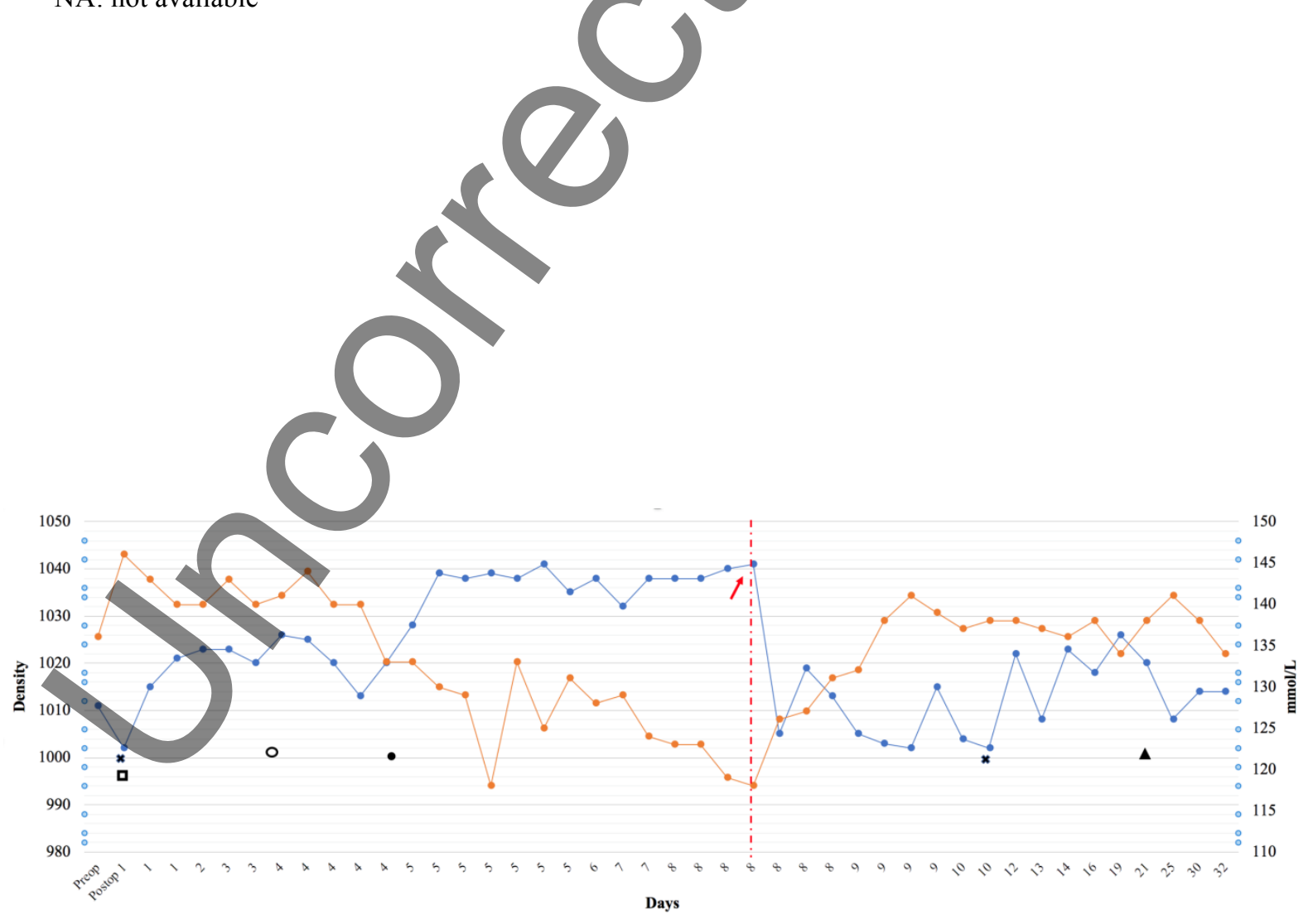

- Urinary Density $\quad \longrightarrow$ Plasma Sodium 
Figure 1. Plasma sodium and urinary output of patient. Cross mark; starting to use desmopressin, square; starting to use dexamethasone, circle; starting to use levothyroxine, point; desmopressin cessation time, arrow mark; tolvaptan treatment, triangular; switching of dexamethasone to hydrocortisone

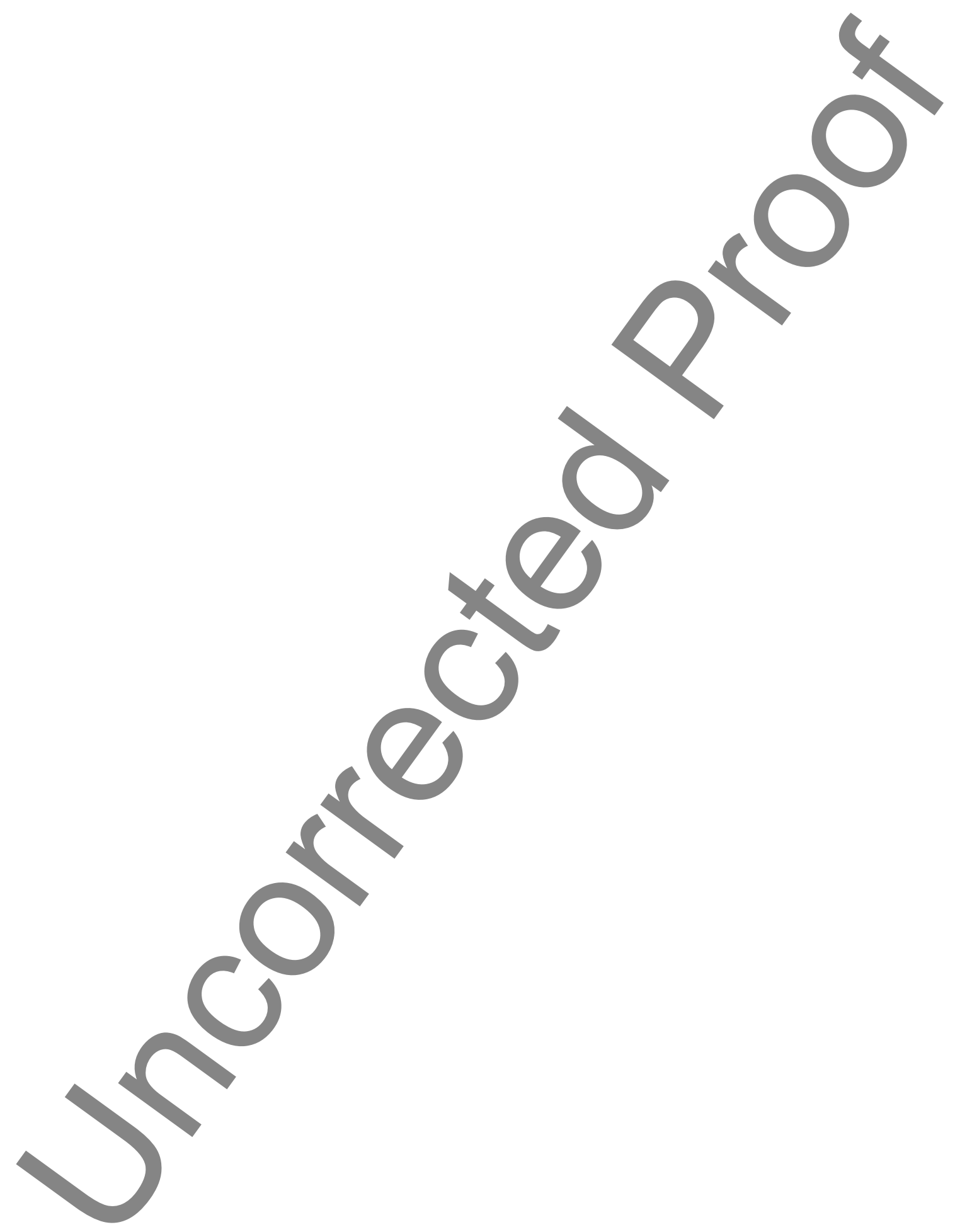

